\title{
USO DE PLANTAS MEDICINAIS PARA TRATAR A MALÁRIA*
}

\author{
Annelita Almeida Oliveira Reiners ${ }^{1}$, Hebert Almeida Ricci ${ }^{2}$, Rosemeiry Capriata de Souza Azevedo ${ }^{3}$
}

\begin{abstract}
RESUMO: Estudo de abordagem qualitativa, cujo objetivo foi investigar o uso de plantas medicinais para tratar a malária por usuários de serviços de saúde pública de um município de Mato Grosso - Brasil. Participaram do estudo 16 pessoas acometidas por malária falciparum ou vivax. Os dados foram coletados por meio de entrevistas semiestruturadas, posteriormente organizadas e analisadas usando a técnica de Análise de Conteúdo. Os resultados mostraram que, no combate à malária, os usuários usam plantas medicinais para complementar o tratamento alopático, pois essas ajudam a aliviar os sintomas da enfermidade e os efeitos secundários dos medicamentos. As plantas mais utilizadas como coadjuvantes foram: boldo, figatil, quina e picão, embora somente a quina e o picão tenham, comprovadamente, propriedades que combatem a malária. Ações educativas sobre o uso adequado e seguro de plantas medicinais,
\end{abstract} considerando crenças e valores culturais, é recomendado.

PALAVRAS-CHAVE: Terapias complementares; Plantas medicinais; Malária.

\section{THE USE OF MEDICINAL PLANTS TO TREAT MALARIA}

\begin{abstract}
A qualitative study which aim was to investigate the use of medicinal plants to treat malaria by users of health services in a municipality of Mato Grosso - Brazil. The participants were 16 people suffering from malaria falciparum and vivax. Data were collected through interviews, then organized and analyzed using the technique of content analysis. The results showed that, in combating malaria, users use medicinal plants to complement allopathic treatment, as these help relieve the symptoms of the disease and the side effects of medications. The plants were being used as adjuncts: Boldo, figatil, cinchona and prick, but only prick and corner have proven properties that combat malaria. Educational activities concerning the proper and safe use of medicinal plants, considering beliefs and cultural values, is recommended.
\end{abstract}

KEYWORDS: Complementary therapies; Medicinal plants; Malaria.

\section{USO DE PLANTAS MEDICINALES PARA TRATAMIENTO DE MALARIA}

RESUMEN: Estudio de abordaje cualitativo, cuyo objetivo fue investigar el uso de plantas medicinales para tratamiento de malaria por usuarios de servicios de salud pública de un municipio de Mato Grosso - Brasil. Han participado del estudio 16 personas acometidas por malaria falciparum o vivax. Los datos fueron recogidos por medio de entrevistas semiestructuradas, posteriormente organizadas y analizadas usando la técnica de Análisis de Contenido. Los resultados mostraron que, en combate a malaria, los usuarios utilizan plantas medicinales para complementar el tratamiento alopático, pues estas ayudan a aliviar los síntomas de la enfermedad y los efectos secundarios de las medicinas. Las plantas más utilizadas como coadyuvantes fueron: boldo, figatil, quina (rubiácea) y picão (Bidens graveolens), aunque solamente la quina y el picão tienen propiedades que combaten la malaria. Acciones educativas sobre el uso adecuado y seguro de plantas medicinales, considerando creencias y valores culturales son recomendadas.

PALABRAS CLAVE: Terapías complementares; Plantas medicinales; Malaria.

*Pesquisa financiada pela Fundação de Amparo à Pesquisa de Mato Grosso-FAPEMAT.

${ }^{1}$ Enfermeira. Doutora em Enfermagem. Docente da Faculdade de Enfermagem da Universidade Federal de Mato Grosso-UFMT.

${ }^{2}$ Enfermeiro da Secretaria Municipal de Saúde de Cuiabá-MT.

${ }^{3}$ Enfermeira. Doutora em Enfermagem. Docente da Faculdade de Enfermagem da UFMT.

Autor correspondente:

Rosemeiry Capriata de Souza Azevedo

Universidade Federal de Mato Grosso

Rua C, 33 - 78048-298 - Cuiabá-MT, Brasil

Recebido: 04/01/10

E-mail:capriata@terra.com.br

Aprovado: 10/06/10 


\section{INTRODUÇÃO}

A malária é uma doença infecciosa, não contagiosa, reconhecida como um grave problema de saúde pública no mundo, ocorrendo principalmente na África, Sudeste Asiático e na Região Amazônica da América do Sul, numa incidência anual estimada em 300 milhões de casos, sendo $80 \%$ deles em países africanos $^{(1)}$. No continente americano, essa doença ameaça a vida de 40 milhões de pessoas ${ }^{(2)}$.

No Brasil, a doença ainda apresenta elevado risco de transmissão na Amazônia Legal, onde aproximadamente $99 \%$ dos casos se concentram devido às condições socioeconômicas e ambientais que favorecem a proliferação do mosquito do gênero Anopheles, vetor da doença. A malária provoca também um impacto socioeconômico significativo por causa dos óbitos, dos dias que o doente deixa de trabalhar, do sofrimento, das perdas sociais, da diminuição dos investimentos empresariais e exploração do potencial turístico dos locais endêmicos.

No ano de 2006, foram notificados 6.245 casos de malária no Estado de Mato Grosso sendo o Município de Colniza o que apresentou maior número deles (3.111 casos), seguido do Município de Aripuanã, com 467 casos e Juína, com 341 casos. No Município de Juruena, 100 casos foram notificados ${ }^{(3)}$.

O tratamento da malária no Brasil consiste em esquemas terapêuticos prescritos pelos profissionais de saúde conforme a determinação contida no Manual de Terapêutica da Malária do Ministério da Saúde lançado, em 2003, pelo Programa Nacional de Prevenção e Controle da Malária (PNCM) da Secretaria de Vigilância em Saúde do Ministério da Saúde ${ }^{(4)}$.

Estudos, realizados principalmente na África, têm mostrado que as populações investigadas, para tratar a malária, utilizam plantas medicinais isolada ou juntamente com a medicação alopática ${ }^{(5-6)}$. Na pesquisa "Motivos para a não-adesão de usuários ao tratamento da malária por Plasmodium Vivax", financiada pela Fundação de Amparo à Pesquisa de Mato Grosso (FAPEMAT), ainda não publicada e desenvolvida em 2007, sobre o comportamento de usuários de um serviço de saúde do Norte do Estado de Mato Grosso em relação ao tratamento da malária, observou-se que os moradores mencionavam o uso de plantas medicinais para combater a doença. Considerando essa prática como parte significativa do cuidado à saúde, em todas as culturas, tivemos interesse em conhecer quais as plantas medicinais que aquela população utiliza para debelar a enfermidade. Além disso, questionamos se as plantas eram usadas como tratamento alternativo ou complementar ao tratamento alopático preconizado pelo Ministério da Saúde.

Desta forma, desenvolvemos esta pesquisa com o objetivo de investigar o uso de plantas medicinais para tratar a malária por usuários de serviços de saúde pública de um município de Mato Grosso.

\section{METODOLOGIA}

Trata-se de um estudo descritivo-exploratório de abordagem qualitativa, realizado com 16 usuários atendidos pelos serviços de saúde pública de um município do Norte do Estado de Mato Grosso, região central do Brasil. Definiram-se como critérios para a seleção dos sujeitos ser usuário da rede básica de saúde, maior de 18 anos, e que declarasse ter contraído malária nos últimos cinco anos. Primeiramente, pediu-se para os Agentes de Saúde da cidade indicar os sujeitos que preenchiam os critérios, depois foi solicitada aos participantes a indicação de outras pessoas que preenchessem os critérios de inclusão no estudo. O tamanho da amostra foi definido usando-se o princípio da saturação dos dados, que prevê que a coleta dos mesmos pode terminar quando nenhuma informação nova é obtida e começa a ocorrer redundância.

A maior parte dos sujeitos do estudo morava em um assentamento onde, nos anos de 2003 e 2004, ocorreu um surto de malária em decorrência da intensa atividade garimpeira desenvolvida no local, naquela época.

Cada participante foi abordado em sua casa pelos pesquisadores que se identificavam, apresentavam o objetivo da pesquisa, seus procedimentos, riscos, benefícios, bem como lhe asseguravam o sigilo das informações e o direito de não mais participar da pesquisa, a qualquer momento. Depois, foi solicitado seu consentimento para realizar a entrevista, pedindo que assinasse o Termo de Consentimento Livre e Esclarecido. A pesquisa foi autorizada pelo Comitê de Ética em Pesquisa da Secretaria de Estado de Saúde, sob o protocolo n. 064/2005/CEP/SES-MT.

A coleta dos dados foi realizada por meio de entrevistas semiestruturadas direcionadas por um roteiro composto de perguntas fechadas e abertas sobre as características dos usuários e sobre o uso de plantas medicinais para tratar a malária. A coleta dos dados 
ocorreu nos meses de agosto e setembro de 2007. Os dados foram organizados e analisados utilizando-se os procedimentos da técnica de Análise de Conteúdo, conforme preconizada por Bardin e, posteriormente, as categorias que emergiram foram discutidas à luz da literatura sobre $\mathrm{o}$ assunto. Os sujeitos da pesquisa foram identificados com E1, E2, e assim consecutivamente.

\section{RESULTADOS E DISCUSSÃO}

Um total de 16 pessoas participaram do estudo, 8 do sexo feminino e 8 do sexo masculino, e com idades entre 19 e 60 anos. A maioria era casada, possuía o ensino fundamental incompleto e tinha como ocupação principal a agricultura familiar. Todos foram acometidos por malária nos últimos quatro anos. Dez dos usuários afirmaram ter contraído malária tanto do tipo falciparum quanto do vivax, enquanto seis deles contraíram apenas a do tipo vivax.

A análise e interpretação dos dados obtidos a partir dos relatos permitiram a elaboração de duas categorias, que mostraram como ocorria o uso de plantas medicinais para tratar a malária.

\section{Plantas medicinais: tratamento complementar da} malária

Os resultados revelaram que a maioria dos usuários pesquisados utilizou como tratamento para a malária o esquema terapêutico preconizado pelo Ministério da Saúde ${ }^{(4)}$, enquanto que uma pequena parcela (dois) fez uso de um tratamento diferente, oferecido por uma instituição religiosa local, como se observa no relato a seguir:

Eu nunca senti o gosto do comprimido da SUCAM [Superintendência de Campanhas da Saúde Pública]. Tratei tudo com o remédio homeopático [da Pastoral da Saúde] e chá caseiro. (E1)

A Pastoral da Saúde é uma organização cívico-religiosa, sem fins lucrativos, que possui atuação de âmbito nacional e de referência internacional. Atua em três dimensões: solidária, comunitária e políticoinstitucional, desenvolvendo, entre outras ações, as de promoção da saúde e prevenção de doenças ${ }^{(7)}$. Em Mato Grosso, existe uma unidade dessa instituição em vários municípios do estado, a qual desenvolve ações de saúde para a população local, inclusive para tratar a malária, utilizando homeopatia.
Tanto os usuários que trataram a malária com o esquema terapêutico preconizado pelo Ministério da Saúde, quanto os que utilizaram o tratamento oferecido pela instituição religiosa, fizeram uso de plantas medicinais (boldo, figatil, quina, picão, entre outras) como forma complementar de tratar a doença. Eles acreditavam que a cura da malária só poderia ocorrer com o tratamento alopático ou homeopático e que as plantas medicinais ajudariam a amenizar os sinais e sintomas da doença e/ou os efeitos colaterais dos medicamentos.

Pergunta - O senhor acha que esses remédios [as plantas medicinais] curam a malária?

Acho que não, mas ajudam a cortar, por exemplo, o sintoma de vômito, e é bom para o rim também. É muito bom. (E3)

Pergunta - O que curou o senhor? As plantas medicinais, o comprimido [antimaláricos] ou o remédio da Pastoral?

Primeiramente, é o que o pessoal da SUCAM passa [antimaláricos], por que corta o vírus da malária; $e$ o outro [as plantas medicinais], a gente toma para desintoxicar o figado. (E15)

[...] são os remédios da malária que fazem a gente sarar. Se a gente não tomar todo o remédio, não sara. (E7)

Resultado semelhante foi encontrado em uma investigação desenvolvida em Uganda, sobre o uso de plantas medicinais no tratamento da malária. Nela, foi constatado que os participantes do estudo afirmaram preferir a medicina alopática às plantas medicinais, principalmente porque consideravam que não tinham conhecimento suficiente sobre as plantas e, ainda, porque acreditavam que os medicamentos alopáticos eram mais eficazes contra a doença $a^{(5)}$.

Tais resultados - somados aos de uma revisão de literatura sobre buscas de tratamento para a doença na qual se identificou um número considerável de estudos que sugeriam que a maioria das pessoas reconheciam o valor das drogas modernas no tratamento da malária ${ }^{(8)}$ - sugerem que os medicamentos antimaláricos têm ganhado credibilidade no tratamento da doença até mesmo entre as pessoas da população.

Como mencionamos anteriormente, os sujeitos do estudo utilizavam as plantas medicinais para com- 
plementar o tratamento da malária, tornando o processo da enfermidade menos doloroso e traumático. A pessoa acometida por malária pode apresentar sinais e sintomas, tanto da doença quanto decorrentes da terapia antimalárica, que provocam um grande desconforto à pessoa doente: mal-estar, cefaleia, cansaço, artralgia e mialgia; anorexia, diarreia, náuseas e vômitos; febre alta $\left( \pm 41^{\circ} \mathrm{C}\right)$ acompanhada de calafrios, sudorese profusa e fraqueza intensa; boca amarga, visão turva, zumbido, tremores e sonolência ${ }^{(9-10)}$.

Tomava o sabugo preto com o picão pra acabar com a malária e o amarelão. Agora, pro figado, quando atacava de novo, ai eu fazia o chá de figatil. (E8)

Quando dava uma prisão de ventre e inchaço e dor na barriga e no fígado. Ai eu tomava [chá de figatil]. (E9)

Esse resultado reforça estudos que têm mostrado que pessoas recorrem ao uso de plantas medicinais para alívio de sinais e sintomas de doenças, bem como para atenuar os efeitos colaterais das terapêuticas a que necessariamente têm que submeter ${ }^{(11-12)}$.

Essa prática baseada no saber popular se integra às práticas dos serviços de saúde trazendo nova conformação a elas ou seja: "esses métodos não foram sufocados pelo saber científico, exatamente porque podem oferecer respostas às enfermidades e sofrimentos vividos pelas pessoas em seu cotidiano"(13:70).

\section{Plantas medicinais utilizadas pelos usuários e suas percepções}

É comum que populações, principalmente em regiões onde os serviços de saúde são deficitários, utilizem plantas medicinais para tratar da saúde por estarem disponíveis a elas em suas casas ou na comunidade, sendo muitas vezes a única alternativa que elas possuem. No caso da malária, estudos referem o uso de plantas medicinais por comunidades em que a doença é endêmica ${ }^{(14-15)}$.

No presente estudo, diversas plantas foram mencionadas pelos usuários como coadjuvantes no tratamento da malária, sendo o boldo (Coleus barbatus Benth), o figatil (Vernonia condensata Backer), a quina (Chinchona calisaya) e o picão (Bidens pilosa L.) as mais citadas.

Chá a gente tomava aqui também. Depois que passava [a malária], tomava. E4
Pergunta - Chá de quê?

Chá de figatil, de boldo. Esse, só às vezes, porque ele é mais amargo. Tem outro também: Zé do Ari. (E4)

Durante o tratamento da malária, usei os remédios da homeopatia e também tomava a quina, o boldo. (E1)

Exceto a quina - que possui um principio ativo que comprovadamente combate a malária, que é a quinina - e também o picão, que tem o princípio ativo fenilacetileno que reduz a parasitemia da malária ${ }^{(16,17)}$, não foram encontrados trabalhos científicos que citassem o boldo e o figatil como plantas propícias ao combate dos efeitos da malária. Em um estudo de revisão das pesquisas desenvolvidas sobre as plantas medicinais usadas para debelar a malária, os autores citaram, entre as várias espécies de plantas estudadas, a quina e a artemísia, mas nenhuma menção foi feita às outras espécies relatadas pelos usuários desta pesquisa ${ }^{(14)}$.

Entretanto, essas plantas têm propriedades que podem ajudar a amenizar a anorexia, a diarreia, as náuseas e os vômitos que a pessoa acometida por malária pode apresentar, contribuindo, de fato, para a melhora do seu estado de saúde.

O boldo estimula o sistema nervoso central e aumenta os movimentos intestinais, sendo que o extrato aquoso da planta tem atividade antidispéptica que reduz a secreção gástrica e protege contra a úlcera gástrica produzida pelo estresse ${ }^{(18)}$. Já o figatil tem ação citoprotetora da mucosa gástrica e as infusões preparadas com as folhas são usadas para o tratamento de cefaleias de origem digestiva ${ }^{(19)}$.

Na medida em que as plantas medicinais são utilizadas pelos usuários, seus efeitos são sentidos gerando percepções de que tais plantas são boas e eficazes para o combate dos sinais e sintomas que eles apresentam.

Remédio do mato sempre é bom, porque ele é amargo e ajuda abrir o apetite. Ele é bom pro estômago que fica embrulhando, dando ânsia de vômito. Remédio amargo segura. (E3)

É bom pra malária, pra limpar o fígado da gente. (E8)

É bom para o figado. Sempre que a gente sente mal, toma um chá que é bom. (E16)

A percepção de que as plantas propiciam a 
recuperação da saúde parece guardar estreita relação com o entendimento que os usuários têm de que elas agem diretamente onde a doença se localiza ou na parte do organismo que ela mais prejudica, geralmente, o fígado.

Pergunta - Por que o senhor tomava esses chás?

Porque se você sente uma dor no figado, porque no figado você sente a malária. Hoje mesmo, se você chupar uma melancia e sentir dor no figado, faz um chá de boldo que acalma, melhora. (E1)

Pergunta - Qual o motivo da senhora tomar este chá?

\section{O figado estava doendo. (E13)}

Na percepção dos usuários sobre a ação das plantas medicinais no organismo, portanto, parece existir um significado ligado à efetividade delas. A planta seria eficaz por agir diretamente sobre os sinais e sintomas, aliviando-os. Essa percepção talvez tenha relação com o conhecimento sobre a doença adquirido pelos usuários a partir do contato com os Agentes de Saúde que, nas orientações fornecidas a eles, apontariam o fígado como um dos locais do corpo onde temporariamente os parasitas se alojam.

Entretanto, é possível que exista na fala dos usuários uma confusão anatômica, pois no conjunto de sinais e sintomas da malária, descrito na literatura, a hepatomegalia e a hipersensibilidade do fígado geralmente não estão presentes. Mas, como sinal visível da doença, o mais comum é que ocorra a esplenomegalia, podendo o baço tornar-se, inclusive, mais sensível. Neste sentido, talvez os usuários, ao mencionarem o fígado como local onde a malária se instalava e se manifestava, na realidade poderiam estar se referindo ao baço, pois, na maioria das vezes, a pessoa leiga não sabe diferenciar a localização do fígado e do baço.

Independente de uma explicação plausível, o que parece evidente é que as plantas medicinais são utilizadas pelos usuários pesquisados apenas como tratamento complementar da malária. Pela percepção de sua eficácia em dirimir os sinais e sintomas sentidos e em atuar diretamente sobre a parte do corpo afetada, são aprovadas por eles e incorporadas ao seu arsenal terapêutico contra a doença.

Isso mostra a importância do saber popular na vivência do processo de saúde-doença das pessoas que, aliado ao conhecimento científico, pode ganhar força e trazer melhor qualidade de vida para elas. Cabe ao enfermeiro, portanto, valorizar esse saber, adequando-o, quando necessário, por meio de atividades educativas com os usuários, "a fim de minimizar ou impedir a ocorrência de casos de intoxicação ou de outros agravos à saúde decorrentes do uso indevido das plantas medicinais"(20:207).

\section{CONCLUSÃo}

Este estudo revelou que, em um município de Mato Grosso, o uso de plantas medicinais pela população pesquisada tinha a finalidade de complementar o tratamento alopático preconizado pelo Ministério da Saúde ou o alternativo desenvolvido por uma instituição religiosa local. O estudo revelou também que a população acredita que o tratamento alopático é mais eficaz para a cura da malária e que as plantas medicinais ajudam a amenizar os sintomas da doença e/ou os efeitos colaterais dos medicamentos.

As plantas mencionadas pelos usuários como coadjuvantes no tratamento da malária, foram o boldo (Coleus barbatus Benth), o figatil (Vernonia condensata Backer), a quina (Chinchona calisaya) e o picão (Bidens pilosa L.). Exceto a quina e o picão, que comprovadamente têm propriedades que combatem a malária, as outras plantas mencionadas podem apenas ter ações sobre os sintomas dispépticos provocados pela doença ou pelos medicamentos.

Os usuários têm a percepção de que essas plantas são boas e eficazes por agir diretamente na parte do organismo que eles entendem ser a mais afetada pela malária, ou seja, o fígado.

Considera-se que este estudo trouxe contribuição para o conhecimento sobre as maneiras que as pessoas lidam com a malária e seu tratamento, bem sobre as plantas medicinais que correntemente são usadas como tratamento complementar da doença, no município matogrossense pesquisado.

Assim como nesse município, outras pessoas, em outros locais do Brasil e do mundo, podem estar usando plantas medicinais no cuidado da malária. Isso remete à necessidade de que novas pesquisas sejam realizadas e que outros aspectos do uso de plantas medicinais no combate à doença sejam explorados.

Por fim, cabe aos profissionais de saúde articular esse conhecimento à sua prática, com o desenvolvimento de ações educativas aos usuários, que promovam a adesão ao tratamento convencional, mas que incluam o uso adequado e seguro das plantas 
medicinais, valorizando as crenças e valores daqueles.

\section{REFERÊNCIAS}

1. Tauil PL. Perspectivas de controle de doenças transmitidas por vetores no Brasil. Rev Soc Bras Med Trop. 2006;39(3):275-7.

2. Organización Panamericana de la Salud. Intensificar lucha contra la malaria. 2005 [Internet] [acesso em 06 dez 2009]. Disponível: http://tiny.cc/3os73

3. Ministério da Saúde (BR). Notificação de Caso/ Ministério da Saúde, Sistema de vigilância de saúde - SVS. Secretaria de Informação de Vigilância Epidemiológica. Brasília; 2007.

4. Ministério da Saúde (BR). Programa Nacional de Prevenção e Controle da Malária - PNCM. Brasília; 2003.

5. Tabuti JRS. Herbal medicines used in the treatment of malaria in Budiope county, Uganda. J Ethnopharmacol. 2008;116(1):33-42.

6. Essé C, Utzinger J, Tschannen AB, Raso G, Pfeiffer C, Granado S et al. Social and cultural aspects of "malaria" and its control in central Côte d'Ívoire. Malar J. [Internet] 2008;7(224). [acesso em $20 \mathrm{dez} 2009$ ]. Disponível: http://tiny.cc/1m3u5. doi:10.1186/14752875-7-224.

7. Oliveira AL. Propostas da Pastoral da Saúde - CNBB para a XII Conferência Nacional de Saúde: "Saúde um direito de todos e dever do Estado - a saúde que temos e o SUS que queremos": [Internet] Conferência Sérgio Arouca. [acesso em $01 \mathrm{fev} 2008]$. Disponível: http://tiny.cc/yga6b

8. McCombie SC. Treatment seeking for malaria: a review of recent research. Soc Sci Med. 1996;43(6):933-45.

9. Ministério da Saúde (BR). Secretaria de Vigilância em Saúde. Ações de controle da malária: manual para profissionais de saúde na atenção básica. Brasília; 2006.

10. Ministério da Saúde (BR). Fundação Nacional da Saúde. Manual de terapêutica da malária. Brasília; 2001.

11. Richardson J. What patients expect from complementary therapy: a qualitative study. Am J Public Health. 2004;94(6):1049-53.

12. Jaconodino CB, Amestoy SC, Thofehrn MB. A utilização de terapias alternativas por pacientes em tratamento quimioterápico. Cogitare Enferm. 2008;13(1):61-6.

13. Siqueira KM, Barbosa MA, Brasil VV, Oliveira LMC, Andraus LMS. Crenças populares referentes à saúde: apropriação de saberes sócio-culturais. Texto \& Contexto Enferm. 2006; 15(1):68-73.

14. Willcox ML, Bodeker G. Traditional herbal medicines for malaria: clinical review. BMJ. 2004; 329(13):1156-9.

15. Kaona FAD, Tuba M. A qualitative study to identify community structures for management of severe malaria: a basis for introducing rectal artesunate in the under five years children in Nakonde District of Zambia. BMC Public Health. [Internet] 2005; 5(28). [acesso em $20 \mathrm{dez}$ 2009]. Disponível: http://tiny.cc/ if0uz

16. Armond C, Casali VWD, Cecon PR, Reis EL, Filho LNC, Lisboa SP et al. Teor de óleo essencial e compostos antimaláricos em plantas de Bidens pilosa L. tratadas com a homeopatia China. Rev Bras Plantas Med. 2005;7(3):18-24.

17. Botsaris AS. Plants used traditionally to treat malaria in Brazil: the archives of Flora Medicinal. J Ethnobiol Ethnomed. [Internet] 2007;3(18). [acesso em 05 mai 2009]. Disponível: http://tiny.cc/vx5qr

18. Fischman LA, Skorupa LA, Souccar C, Lapa AJ. The water extract of Coleus barbatus Benth decreases gastric secretion in rats. Mem Inst Oswaldo Cruz. 1991;86 (Suppl 2):S141-3.

19. Lolis MIGA, Milaneze-Gutierrez MA. Morfoanatomia das folhas de Vernonia condensata Baker (Asteraceae), o "figatil". Rev Bras Farmacogn. [Internet] 2003;13 (Supl). [acesso em 06 jan 2008]. Disponível: http://tiny.cc/q1fox

20. França ISX, Souza JA, Baptista RS, Britto VRS. Medicina popular: benefícios e malefícios das plantas medicinais. Rev Bras Enferm. 2008;61(2):201-8. 